\title{
Search the optimal ratio of system's component reliability parameters and the heat capacity of prosumers in the district heating system
}

\author{
Valery Stennikov ${ }^{1}$, and Ivan Postnikov ${ }^{1, *}$ \\ ${ }^{1}$ Melentiev Energy Systems Institute of Siberian Branch of the Russian Academy of Sciences, \\ Laboratory of Heat Supply Systems, 664033 Irkutsk, Russia
}

\begin{abstract}
The paper presents a methodology for ensuring the reliability of heat supply to prosumer connected to a district heating system, by using the heat capacity of its own heat source and optimization of failure rates of system components. To solve this problem, a mathematical formalization is developed, within which a markov random process, a flow distribution model of the theory of hydraulic circuits, nodal reliability indices, and simplified models of heat transfer processes in heat supply to consumers are used. A case study is considered based on a test scheme of district heating system with prosumer, an analysis of the results obtained is carried out, conclusions and directions for the further research on the subject are formulated.
\end{abstract}

Keywords: district heating system; prosumer; mathematical modeling; optimization; failure rate; nodal reliability indices; markov random process; theory of hydraulic circuits.

\section{Introduction}

The development of modern energy systems is the implementation of new efficient and low emission technologies, their transition to new principles of designing and management based on the integration of different energy technologies, and the using of intelligent and optimal system of operating of technological and economical processes [1-2]. In this systems, as a rule, so called prosumers are used which optimally regulate their heat consumption schedule by own energy source and/or energy storages, decreasing the load on district sources and network (for example for the peak of load).

In the present time there is a lot of researches on methodological and technical aspects of the implementation of prosumer concept in electric power systems (EPS), some works on this are presented in [4-8]. The use of prosumers in district heating systems (DHS) is given much less attention, although this technology is no less (possibly more) relevant for this systems, which has a much more potential for energy saving compared to EPS, especially in the countries with a cold climate. Nevertheless, in recent years, attention to the issue of prosumer in DHS has increased significantly, including the transition to a new technological level of district heating of the 4th generation (4GDH) [9-11]. For example, in

* Corresponding author: postnikov@isem.irk.ru 
[12-14], the authors present methodological and practical studies on the participation of prosumers in district heating in Sweden and Norway. Further in the paper a prosumers of heat energy connected to DHS is considered.

In the previous studies [15-18] a model for optimal operating of the DHS that involve prosumers with owned heat sources (HS) is proposed. Own HS of prosumer provides an additional heat energy capacity and time redundancy, which allows to reduce heat undersupply in failure states of the system, as well as reduce the load on district/centralized HS and improve quality and reliability of heating for both consumers (including prosumers) and DHS in general. This study is devoted to the development of methods for ensuring the reliability of heat supply to prosumers taking into account their additional redundancy functions by its own HS capacity. The solution of statement problem is search for the optimal relation of reliability parameters of system's components and the active redundancy of prosumer, which is provided by its own generation of thermal energy.

\section{Methods}

The considered problem is the determination of the optimal relation of values of reliability parameters of DHS components (failure rates and/or restoration rates) and the capacity and time redundancy of the prosumer, that ensure the required reliability level of heat supply to prosumers and also other consumer in the system at minimum costs on the system reliability and the operating of prosumer's HS within feasible ranges of parameters and conditions. The solution of the statement problem is carried out using of the integrated reliability parameter of system components. The integrated reliability parameter of components is taken to mean their failure rate or restoration rate that preliminarily has the same value for these components, which provides the required (standard) level of reliability indices (RI). Requirements to reliability of heat supply to consumers can be set on the basis of different RI [19]. In the framework of this study, one of the main comprehensive indices are used: failure-free operation probability (FOP) and availability factor (AF).

An objective function is minimization of the sum of the costs of ensuring the reliability of heating to prosumer both by increasing of system components reliability and by providing additional capacity redundancy of its own HS:

$$
Z_{\mathrm{obj}}=\sum_{j \in J}\left[f_{j}\left(\Delta \varepsilon_{j}\right)+f_{j}\left(q_{j}^{\prime}\right)\right]+\sum_{n \in N} f_{n}\left(\lambda_{n}\right) \rightarrow \min ,
$$

where $j$-number of consumer/prosumer (further consumer means both as consumer and prosumer); $J$ - set of consumers including prosumers; $n$ - number of system component; $N$ - complete set of system components; $f_{j}\left(\Delta \varepsilon_{j}\right)$ - cost function of ensuring additional passive time redundancy of prosumers (by heat storages or/and thermal accumulation effect of own HS), $\$ ; f_{j}\left(q_{j}^{\prime}\right)$ - cost function of ensuring additional active time redundancy of prosumers (by capacity of own HS), $\$ ; f_{n}\left(\lambda_{n}\right)$ - cost function of ensuring failure rates of system components by its redundancy, using of more reliable equipment, designing of more reliable structure of technological nodes, etc., $\$ ; \Delta \varepsilon_{j}$ - additional time redundancy of prosumer $j$ caused by the use of own HS and/or storages, h; $q_{j}^{\prime}$ - heat capacity of own HS for prosumer $j, \mathrm{GJ} / \mathrm{h} ; \lambda_{n}$ - failure rate of component $n, 1 / \mathrm{h}$. It should be noted that within the framework of this research, the increase in reliability due to restoration processes is not considered; therefore, the objective function (1) does not include the costs on the providing of restoration rates of components and problem of reliability optimization is 
solved with respect to costs of achieving the required failure rates in combination with the necessary operating costs for prosumer's HS. This is due to the issue of ensuring the required level of restoration time is a separate problem associated with equipping of emergency teams and developing an optimal system for identifying accidents. Some points on this issue are considered in [20].

Following expressions is determined the integrated failure rate of system components when performing the required levels of RI - AF and FOP:

$$
\begin{gathered}
\bar{\lambda}_{j}=\frac{\ln \left(1 / R_{\mathrm{o} j}\right)}{\tau_{\mathrm{o}}}\left(\left[1-S\left(1-K_{\mathrm{o} j}\right)\right] \sum_{s \in E} M_{s}^{\sigma j}\right)^{-1} \sum_{s \in E} L_{s}^{\sigma j}, \\
M_{s}=\left(\frac{1-\bar{q}_{s j}+\varphi_{j} t_{s j} / q_{\mathrm{o} j}}{1-\omega_{j}}\right)-\left(\frac{\varphi_{j}\left(C_{1}-C_{2} \exp B_{j}\right)}{C_{3} q_{\mathrm{o} j}\left(1-\exp B_{j}\right) 1-\omega_{j}}\right), \\
L_{s}=\left(\frac{1-1 / q_{\mathrm{o} j}}{1-\omega_{j}}\right) \times\left(q_{s j}+\varphi_{j} t_{s j}-\varphi_{j} \frac{C_{1}-C_{2} \exp B_{j}}{C_{3}\left(1-\exp B_{j}\right)}\right), \\
C_{1}=t_{\mathrm{o} j}\left(1-\bar{q}_{s j}\right), C_{2}=t_{j \min }-t_{\mathrm{oj} j} \bar{q}_{s j}, C_{3}=1-\bar{q}_{s j}, \\
\bar{q}_{s j}=q_{\mathrm{o} j} /\left(q_{s j}^{\mathrm{sys}}+q_{j}^{\prime}\right), \\
B_{j}=1 /\left[\left(\varepsilon_{j}+\Delta \varepsilon_{j}\right) \bar{\mu}_{j}\right],
\end{gathered}
$$

where $\bar{\lambda}_{j}$ - integrated (average) failure rate of system components relation to consumer $j$, $1 / \mathrm{h} ; K_{\mathrm{o} j}, R_{\mathrm{o} j}$ - standard level of nodal (for consumer $j$ ) AF and FOP respectively; $\tau_{\mathrm{o}}-$ time instant corresponding to a total number of hours of the considered (heating) period, h; $S$ - number of system states; $s, z$ - numbers of system states; $E$ - complete set of system states; $\omega_{j}, \sigma_{j}$ - irregularity factors of heat load curve of consumer $j[20] ; \bar{q}_{s j}$ - relative heat supply to consumer $j$ in system state $s, \mathrm{GJ} / \mathrm{h} ; \varphi_{j}$ - coefficient of specific heat losses for consumer $j, \mathrm{GJ} /\left(\mathrm{h}^{\circ} \mathrm{C}\right) ; t_{s j}$ - current (actual) internal air temperature for consumer $j$ in system state $s,{ }^{\circ} \mathrm{C} ; q_{\mathrm{o} j}$ - design (maximum) heat load of consumer $j$, $\mathrm{GJ} / \mathrm{h} ; q_{s j}$ - level of heat supply to consumer $j$ in system state $s, \mathrm{GJ} / \mathrm{h} ; t_{j \text { min }}-$ minimum admissible temperature of internal air for consumer $j,{ }^{\circ} \mathrm{C} ; t_{\mathrm{o} j}-$ design temperature of internal air for consumer $j,{ }^{\circ} \mathrm{C} ; q_{s j}^{\text {sys }}$ - part of heat load of consumer $j$, supplied from the system (by district HS) in system state $s, \mathrm{GJ} / \mathrm{h} ; \varepsilon_{j}$ - heat accumulating coefficient for consumer $j, \mathrm{~h} ; \bar{\mu}_{j}$ - integrated (average) restoration rate of system components relation to consumer $j, 1 / \mathrm{h} ; L_{s}, M_{s}, C_{1}, C_{2}, C_{3}, B_{j}$ - assumed abbreviations of expressions. These dependences (2)-(7) is developed using three components: 1) expressions for the calculation of corresponding nodal RI (AF and FOP) for DHS, presented in [19]; 2) Rossander's equations for determining the heat load of each consumer (including prosumers) at any time during the calculating period (usually the heating season period) [20]; 3) formula for calculating the such external temperature at which the time redundancy of consumer (including prosumer) corresponds to the restoration time of the heating of consumer after its interruption, identified by the system state $s$ [19], taking into account 
the total time redundancy due to both the passive heat accumulating effect of buildings and the active capacity redundancy of its own HS or heat storages.

The distribution of the integrated values of reliability parameters among system components is carried out according to follow equalities for failure and restoration rates:

$$
\begin{aligned}
& \bar{\lambda}_{j} \sum_{s \in E} p_{s}=\sum_{n \in N} \sum_{s \in E_{n}} \lambda_{n} p_{s}, \\
& \bar{\mu}_{j} \sum_{s \in E} p_{s}=\sum_{n \in N} \sum_{s \in E_{n}} \mu_{n} p_{s},
\end{aligned}
$$

where $p_{s}$ - probability of system state $s ; \mu_{n}$ - restoration rate of component $n, 1 / \mathrm{h} ; E_{n}$ - subset of system states for which the system can transition because of failure or restoration of component $n$.

Probabilities of each system state is determined from solving the equation system of the stationary markov process describing the evolution of events and states in the system. Applied of markov models for the solving of different reliability problems of DHS in more detail is considered in papers [21-23]. The levels of heat supply to each consumer (incl. prosumers) in different system states (in case of failures in the system components) are determined from calculation of flow distribution in heat network of DHS. The flow distribution (acceptable hydraulic modes) is calculated using the mathematical methods developed within the theory of hydraulic circuits [24], partially, the nodal matrix form of the flow distribution model.

Thus, the problem of optimal relation the time redundancy of prosumer and failre rates of components in DHS lies in the following: minimize function (1) under conditions (2)-(7) and (8), (9) into account constrains for acceptable hydraulic modes in heat network and available values of variables of considered problem. The mathematical method of solving the formulated problem is determined mainly by the form of costs functions including in the objective function (1). Usually, using the approximation methods, these functions can be represented in the form of power dependencies.

\section{Brief report on case study}

The considered scheme of DHS is shown in Fig. 1-a. It has the one district HS as combined heat power plant (CHPP) with an output of $135 \mathrm{GJ} / \mathrm{h}$ and the heat network with a looped part that unites 9 consumers, including the prosumer - node 3 .

In total, 9 consumers are connected to the system (nodes 1-3, 7-10 and 12, 13) with summary load equal $131.6 \mathrm{GJ} / \mathrm{h}$. Heat network and consist of 14 sections (branches) with their characteristics indicated in the Fig. 1-a and by total length equal $1150 \mathrm{~m}$. The aggregated DHS scheme for reliability analysis is presented on Fig. 1-b formed on the basis of initial general scheme (Fig. 1-a) and needed for constructing a set of system states, a graph of states and the corresponding equations system of markov model describing the random process of functioning of the system in the time. In this scheme (Fig. 1-b) numbers $1 *-4 *$ are related to aggregated technological components of district HS.

The conditions for a modeling describing possible failures in the system are determined by the stationary markov random process, according to which reliability parameters of components do not change over time and simultaneous failures possible only for two components of different subsystems of the DHS - district HS or heat network. The compliance of these conditions (stationary and ordinary in the framework for single subsystem) to the actual systems are well confirmed many researches [19]. 

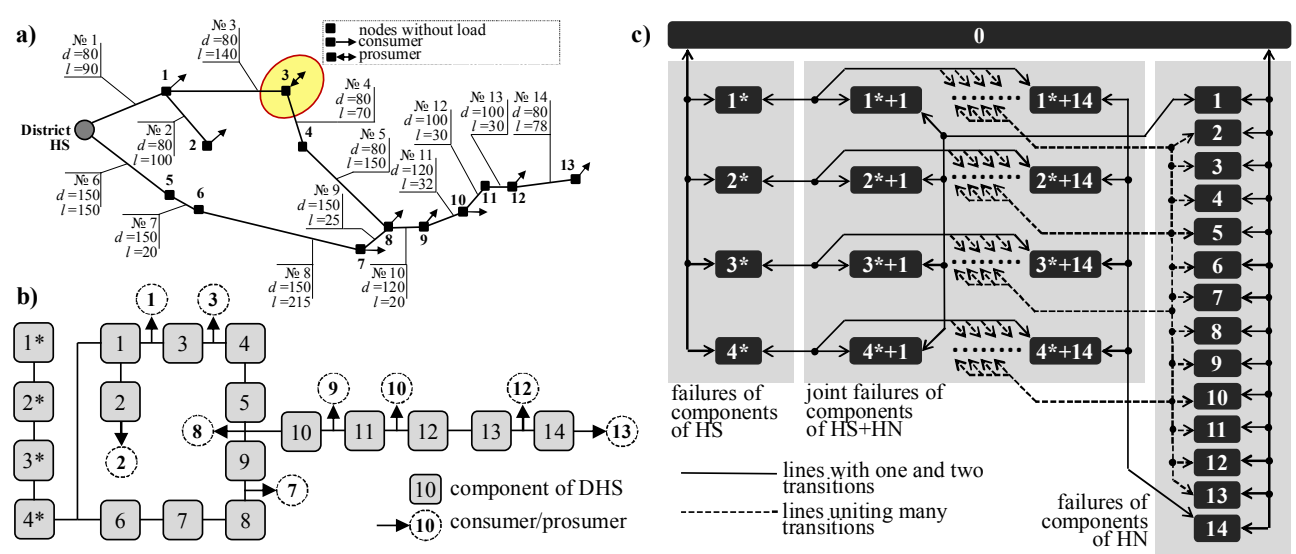

Fig. 1. Schemes for the case study: a) general calculated scheme of DHS: in the callouts $d$ - diameter of a network section, mm; 1 -length of a network section, $\mathrm{m}$; b) calculated scheme of DHS for its reliability analysis; c) a graph of DHS states and transitions between them in aggregated form (corresponds to the scheme of Fig. 1-b)
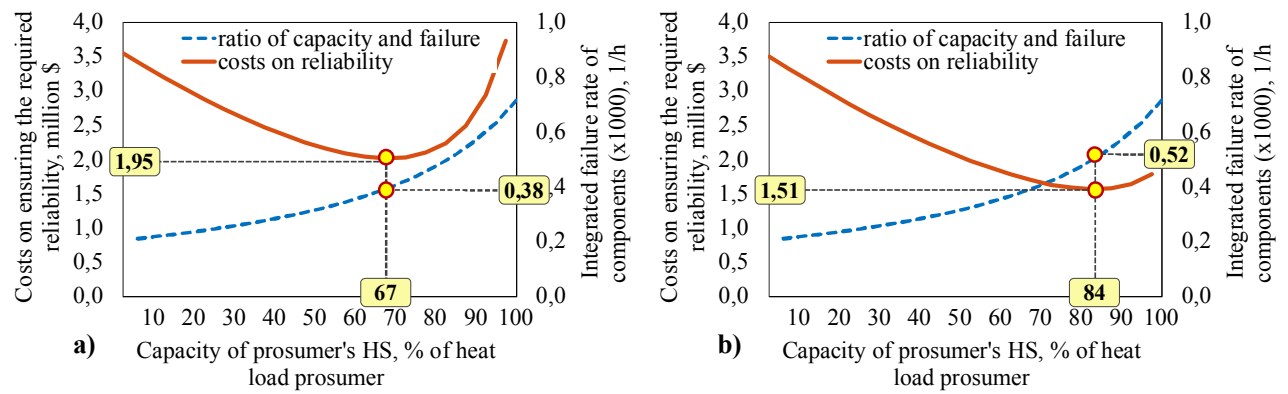

Fig. 2. Search for an optimal relation the capacity of prosumer's HS and failure rates of components in DHS for ensuring of the required reliability level: a) for standard FOP equal 0.92 ; b) for standard FOP equal 0.90

The graph of states and transitions between them, corresponding to the conditions described above, is presented in an abbreviated form in Fig. 1-c. It presents three groups of states: failures of HS components, failures of heat network (HN on the figure) components, joint failures of this components.

The objective function (1) was determined as the sum of power dependencies estimated from the analysis of data on costs on duplication of DHS components (mainly sections of the heat network as less reliable components compared to HS equipment), costs of replacing them with more reliable components, operating costs of HS of prosumer. in different functioning modes (in this case the mini-CHPP running on fossil fuel is considered). The results of calculations carried out on the methodology described above are presented in Fig. 2 for two standard levels of FOP -0.92 and 0.90 .

For example, in order to ensure a higher level of reliability of heat supply to prosumer, corresponding to an FOP equal 0.92 (which means that the interruption frequency of heat supply to consumers is 0.081 /year or 1 time in 12 years), the value of the integral failure rate of components should be no more than $0.38 \mathrm{1} / \mathrm{h}$ while the capacity of prosumer's own HS is $67 \%$ of its heat load. The total cost of ensuring reliability in this case is 1.95 million\$ (Fig. 2-a). The results for the FOP equal 0.90 (which means that the interruption frequency of heat supply to consumers is 0.11 /year or 1 time in 9.5 years) are presented on (Fig. 2-b): the total cost of ensuring reliability in this case is 1.51 million $\$$. 


\section{Conclusions}

The research is dedicated to solving one of the many problems in the field of analysis and synthesis of the reliability of heat supply to consumers in conditions the development of DHS of 4th generation in the framework of general paradigm of the transition to intelligent integrated energy systems. Considered reliability problems, in turn, are a significant part in the formation of a comprehensive methodology for the development of optimal decisions for the designing, operation and development of intelligent and integrated DHS using new energy-efficient technologies, one of which is the prosumer concept.

The research was performed in the framework of a scientific projects III.17.4.3 №AAAA-A17$117030310437-4$ of the foundation researches program of SB RAS, and was funded by RFBR, project number 20-08-00488 A

\section{References}

1. H. Lund, P. Østergaard, D. Connolly, B. Mathiesen, Energy, 137, 556 (2017)

2. P. Mancarella, G. Andersson, J. Peças-Lopes, K Bell, R.W. In: Power Systems Computation Conference (PSCC, Genoa, Italy, 2016)

4. L. Perkovic, H. Mikulcic, N. Duic, Journal of Cleaner Production, 167, 1438 (2017)

5. H. Yang, T. Xiong, J. Qiu et al. Appled Energy, 167, 353 (2016)

6. N. Zhang, Y. Yan, W. Su, Applied Energy, 154, 471 (2015)

7. R. Zafar, A. Mahmood, S. Razzaq et al. Renewable and Sustainable Energy Reviews, 82, $1675(2018)$

8. S. Werner, Energy, 137(15), 617 (2017)

9. H. Lund, S. Werner, R. Wiltshire, et al. Energy, 68, 1 (2014)

10. H. Lund, N. Duic, P. Østergaard, B. Mathiesen, Energy, 110, 1(2016)

11. H. Lund, P. Østergaard, M. Chang, et al. Energy, 164, 147 (2018)

12. L. Brange, J. Englund, P. Lauenburg, Applied Energy, 164, 492 (2016)

13. L. Brand, A. Calvén, J. Englund et al., Applied Energy, 129, 39 (2014)

14. H. Kauko, K. Kvalsvik, D. Rohdeb, et al., Energy, 151, 261 (2018)

15. I. Postnikov, V. Stennikov, A. Penkovskii, Energy Procedia, 158, 2530 (2019)

16. I. Postnikov, V. Stennikov, A. Penkovskii, E3S Web of Conferences, 58 (2018)

17. V. Stennikov, I. Postnikov, A. Penkovskii, E3S Web of Conferences, 25 (2017)

18. I. Postnikov, Energy Reports, 6(2), 214 (2020)

19. E. Sennova, A. Smirnov, A. Ionin et al. Handbook on reliability of heat supply systems (Nauka, Novosibirsk, 2000) [In Russian]

20. E. Sennova, V. Sidler, Mathematical modeling and optimization of developing district heating systems (Nauka, Novosibirsk, 1985) [In Russian]

21. I. Postnikov, V. Stennikov, E. Mednikova, A. Penkovskii, Applied Energy, 227, 365 (2018)

22. V. Stennikov, I. Postnikov, Power Technology and Engineering, 47(6), 446 (2014)

23. I. Postnikov, V. Stennikov, Energy Reports, 6(2), 293 (2020)

24. A. Merenkov, V. Khasilev, Theory of hydraulic circuits (Nauka, Moscow, 1985) [In Russian] 